\title{
A novel detection strategy for optical receivers with focal plane array
}

\section{Kamran Kiasaleh}

Kamran Kiasaleh, "A novel detection strategy for optical receivers with focal plane array," Proc. SPIE 11852, International Conference on Space Optics ICSO 2020, 118525H (11 June 2021); doi: 10.1117/12.2599960 SPIE. Event: International Conference on Space Optics - ICSO 2021, 2021, Online 


\section{International Conference on Space Optics-ICSO 2020}

Virtual Conference

30 March-2 April 2021

Edited by Bruno Cugny, Zoran Sodnik, and Nikos Karafolas
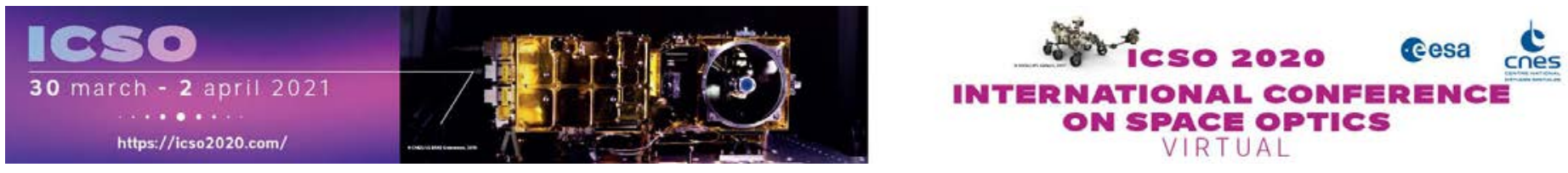

\section{A Novel Detection Strategy for Optical Receivers with Focal Plane Array}

\section{Cesa isoporoceatings denes}




\title{
A Novel Detection Strategy for Optical Receivers with Focal Plane Array
}

\author{
Kamran Kiasaleh*a \\ ${ }^{a}$ The University of Texas at Dallas, \\ Erik Jonsson School of Engineering and Computer Science, \\ 800 W. Campbell Rd., Richardson, TX, USA
}

\begin{abstract}
In this paper, the problem of optimizing the performance of a free-space optical system with large field of view (FOV) focal plane array is addressed. A large FVO detector is desired when beam tilt, large pointing inaccuracies, or other impairments leading to uncertainties in the angle of arrival of the beam are present. For such an arrangement, when we consider a large aperture size, which results in a small diffraction-limited FOV, a large number of photo-detectors in the array are exposed to background radiation only. In addition, in adaptive optics system without a wave-front sensor (WFS), one has to contend with the same issue when large FOV system with small diffraction-limited FOV is considered. Hence, detection strategies to deal with the excess background noise in this scenario is of interest in this paper. We assume here that the receiver is utilizing a recently proposed technique to identify the presence and the location of the beam footprint in the focal plane, thereby reducing the "area" of consideration in the focal plane to a subset of the total photodetectors. It is shown here that, when the proposed receiver is utilized, one can expect an improvement in the overall bit error rate of several orders of magnitude as compared with the standard array detector.
\end{abstract}

Keywords: Array, Detectors, FSO

\section{INTRODUCTION}

Array detectors can offer enhanced performance in free-space optical (FSO) channels impaired by pointing inaccuracies and other scenarios, which lead to uncertainty in the direction of arrival of optical beams. Such uncertainties may be due to atmospheric tilt or spacecraft/receiver motion or orientation ${ }^{1-11}$. To elaborate, array detectors enable an FSO receiver to compensate for the misalignment at the receiver and capture optical fields that are extended beyond the diffraction-limited field of view of the receiver. To achieve the benefits stated above, the receiver must consider the photodetector (PD) outputs of an array of photodetectors and combines them to achieve the desired performance. A simple combining involves the sum of the detected signals, which we will referred to as the equal gain combining (EGC) method in this paper. Such an approach is reasonable when a small number of detectors are utilizing. For a large array of detectors (we note that, as one considers high data rates, the photodetector area reduces, leading to a large number of required photodetectors in a given detector area), in order to avoid summing a large number of photodetector outputs that contain only background noise, one has to consider maximum ratio combining (MRC) technique. In that event, one must estimate the signal intensity for each photodetector using various techniques proposed in the literature ${ }^{4,5,7}$. Such techniques result in an unreasonable receiver complexity and prohibitive cost when the number of photodetectors are large and one is operating in multi giga bits-per-second (Gbps) environment. For this reason, one must consider a more intelligent approach to combining the output of photodetectors in large detector arrays. One approach is to estimate the presence of the desired optical field in one or several photodetectors and only consider the outputs of such detectors ${ }^{3}$. However, such a technique also requires that one performs estimation of the signal levels ${ }^{4,5,7}$. Such estimation techniques, as noted, are prohibitively complex and can lead to an unacceptable level of complexity in Gbps communications systems. In a recent study, we considered the use of nonlinear processing of the detector outputs without channel estimation ${ }^{9}$. However, the proposed technique requires nonlinear processing of all the outputs of the photodetectors. Hence, the receiver complexity remains high.

In this paper, we propose a simple and yet effective means for combining the detected signals in a detector array. We propose to utilize the technique that we proposed recently to identify the location of the beam within the receiver focal 
plane ${ }^{10}$. We propose to use this algorithm to confine the summation in EGC to only a few PD outputs, which are identified as having been exposed to the received optical beam in the focal plane. Hence, the new technique does not require estimating the signal intensities of a large number of photodetectors and does not suffer from combining a large number of photodetector outputs that contain noise only. Given that signal estimation is not required, the proposed receiver is well suited for deployment in a practical FSO systems operating in high-speed optical communications environments where detector arrays are used.

This paper is organized as follows. In Section 2, we present a possible detector arrangement and describe the optical detection mechanism. Furthermore, the propose method for combining the outputs of the photodetectors is introduced in this section. In Section 3, we present analytical results and discuss the performance of the standard receiver with array detectors and that of the proposed receiver. In Section 4, using numerical analysis, we compare the performance of the standard receiver with that of the proposed receiver under variety of conditions, including when the algorithm used to identify the location of the optical beam within the focal plane is yielding false detection.

Finally, in Section 5, we present the concluding remarks.

\section{SYSTEM MODEL}

\subsection{Detector Array Receiver}

We consider an optical front-end that will capture the received optical field using a lens of diameter $D$ and focal distance of $f$. For an optical field of wavelength $\lambda$, the optical frontend (with a circular aperture of diameter $D$ ) has a diffraction limited field of view proportional to $\frac{\lambda^{2}}{D^{2}}$, which can be designed to meet the system requirement using the proper aperture lens diameter ${ }^{12}$. We assume that the received optical beam is mapped by the optical frontend onto an area of $A_{D}=$ $f^{2} \Omega_{F O V}$ in the focal plane, see Fig. 1. Furthermore, it is assumed that the photodetector area is considered to be $A_{P D}$. Without the loss of generality, we assume that $A_{D}=N^{2} A_{P D}$ with $N^{2} \geq 1$. This implies that the beam footprint illuminates $N^{2}$ photodetectors in the focal plan. Furthermore, the optical assembly using a detector array provides a field of view $\Omega_{A}=$ $\frac{A}{f^{2}}$. For a square shape detector area of $M^{2}$ photodetectors (the receiver can detect $\frac{M^{2}}{N^{2}}$ spatial modes), we have

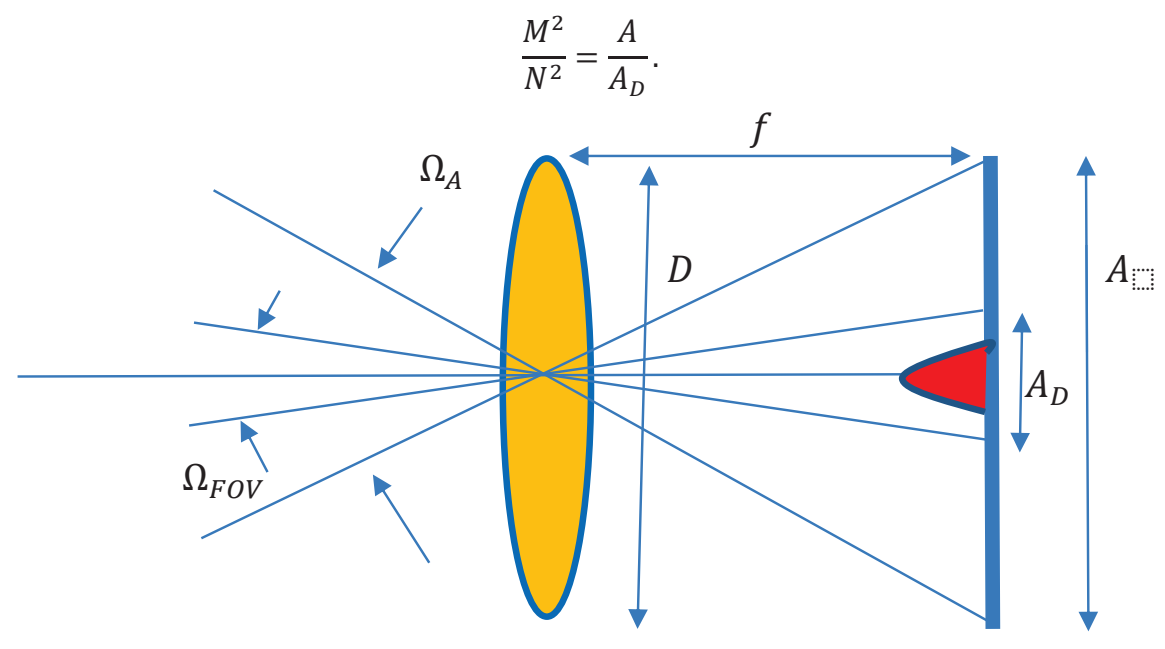

Fig. 1. Focal plane detector array.

As can be seen, this optical assembly is capable of receiving $\frac{M^{2}}{N^{2}}$ spatial modes. This allows for a wide variation in the direction of arrival (beam tilt) as well as pointing jitter and uncertainly. Long-distance optical channels, such as ground to 
space or space to ground laser communications, often suffer from beam broadening at the receiver due to turbulenceinduced beam wander. Such impairment can also be alleviated using the wide field of view array detector shown in Fig. 1.

In what follows, we consider a binary pulse-position modulation (BPPM) and direct-detection at the receiver.

\subsection{Standard Detection Mechanism}

We first present a detection mechanism refereed to here as the standard receiver (SR) where the outputs of the $M^{2}$ photodetectors are used to render a data decision. For the problem at hand, the detectors produce independent signals. Let $r_{i j}^{+}$with $i, j \in[1, M]$ denote the photon count for the $i j$ th photodetector over the $1^{\text {st }}$ slot duration of the binary pulse-position modulation symbol. Further, let $r_{i j}^{-}$denote the similar count for the $2^{\text {nd }}$ time slot of the BPPM signal. Then, the following decision variables are formed:

$$
\Lambda_{1, S R}=\sum_{i=1}^{M} \sum_{j=1}^{M} r_{i j}^{+}
$$

and

$$
\Lambda_{2, S R}=\sum_{i=1}^{M} \sum_{j=1}^{M} r_{i j}^{-}
$$

Then, the optimum receiver is given by

$$
\hat{d}_{S R}=\arg \max \left\{\Lambda_{j, S R}\right\} ; j=1,2,
$$

where $\hat{d}$ denotes the estimate of the transmitted binary data $d \in[0,1]$.

\subsection{Proposed Detection Mechanism}

In this paper, we propose a detector that considers only a subset of $M^{2}$ photodetectors, thereby reducing the impact of background noise.

We assume here that the receiver is utilizing a recently proposed technique to identify the presence and the location of the beam footprint in the focal plane, thereby reducing the "area" of consideration in the focal plane to a subset of the total area occupied by the photodetectors ${ }^{10}$. In another method, as noted in subsection 2.1, a simple sum of the outputs of all photodetectors is considered. This method is referred to as SR. In the SR method, we consider photon counts over every photodetector in the array. We consider a binary pulse-position modulation where the bit rate is $R_{b}=1 / T_{b}$ bits per second.

Hence, we have a BPPM slot with the duration of $T_{s}=\frac{T_{b}}{2}$ sec. For the proposed receiver (PR), we form the following decision variables:

$$
\Lambda_{1, P R}=\sum_{i, j \in \Omega} r_{i j}^{+}
$$

and

$$
\Lambda_{2, P R}=\sum_{i, j \in \Omega} r_{i j}^{-}
$$

where $\Omega$ describes a subset of 2-tuples that identify the location of the beam footprint in the focal plane. Note that $\Omega$ may contain up to $N^{2}$ coordinates as a beam may illuminated up to $N^{2}$ photodetectors. Then, the optimum receiver is given by

$$
\hat{d}_{P R}=\arg \max \left\{\Lambda_{j, P R}\right\} ; j=1,2,
$$


where $\hat{d}_{P R}$ denotes the estimate of the transmitted binary data $d \in[0,1]$ for the PR.

\section{ANALYTICAL RESULTS}

Let the array consist of $M^{2}$ photodetectors $(M \times M$ detectors). Also, let us assume that the received optical beam images onto $N^{2}$ photodetectors. It is assumed that $N^{2} \leq M^{2}$. For a large photodetector array, $M^{2} \gg N^{2}$. Let us also assume that the background radiation results in an average photon count of $K_{b}$ per BPPM slot for each photodetector. Also, let the average signal count per BPPM slot for each photodetector be $K_{s}$ photons. Let $N^{2}$ be the number of photodetectors that are illuminated by the signal portion of the received optical field.

We assume that the level of background noise is large enough to allow for a Gaussian approximation of the statistics of the decision variables shown above. In particular, since a sum of photodetector outputs $\left(M^{2}\right.$ or $\left.N^{2}\right)$ are considered, the Gaussian approximation seems to be a reasonable assumption.

\subsection{Decision Variable Statistics}

We consider a direct-detection receiver operating under the impact of a non-negligible background radiation. We assume a scenario where the output current of the photodetector is integrated for the two consecutive time slots of the BPPM symbol to form $r_{i j}^{+}$and $r_{i j}^{-}$, respectively. Assuming that the $i j$ th photodetector is illuminated by the received radiation, then for a direct-detection receiver, subject to background noise and thermal noise, the expected value of the observed photon count (integrated over a slot duration of a BPPM symbol) when conditioned on the data is given by ${ }^{12}$

and

$$
m_{i j}^{+}=E\left\{r_{i j}^{+} \mid d\right\}=\bar{g} e\left(d K_{s}+K_{b}\right)
$$

$$
m_{i j}^{-}=E\left\{r_{i j}^{-} \mid d\right\}=\bar{g} e\left(d_{c} K_{s}+K_{b}\right)
$$

where $d \in[0,1]$ and $d_{c}$ is the complement of $d$. Furthermore, $e$ and $\bar{g}$ denote the charge of an electron and the average gain of the photodetector (this includes the amplifier gain that will be used to amplify the detected signal), respectively. Hereafter, overbar signifies the ensemble average. Furthermore, the variances of $r_{i j}^{+}$and $r_{i j}^{-}$, respectively, are given by ${ }^{12}$

$$
\sigma_{+, i j}^{2}=F \bar{g}^{2} e^{2}\left(d K_{s}+K_{b}\right)+\sigma_{n}^{2}
$$

and

$$
\sigma_{-, i j}^{2}=F \bar{g}^{2} e^{2}\left(d_{c} K_{s}+K_{b}\right)+\sigma_{n}^{2}
$$

where $F$ denotes the photodetector's excess noise factor. For p-i-n photodetectors, typically $F=1$. For avalanche photodetectors, however, $F>1$. Furthermore ${ }^{12}$,

$$
\sigma_{n}^{2}=\frac{2 k T_{0}}{R_{L}} T_{s}
$$

is the variance of the thermal noise contribution to the photon count where $k, T_{0}$, and $R_{L}$ denote the Boltzmann's constant, the operating temperature of the receiver in Kelvin, and the load resistance of the photodetector in ohms. Furthermore, $T_{S}$ denotes the BPPM slot duration in sec.

\subsection{Bit Error Analysis}

Given the above formulation and the fact that the photodetectors' outputs form independent random variables, the mean values of the decision variables are given by

and

$$
\bar{\Lambda}_{1, P R}=N^{2} \bar{g} e\left(d K_{s}+K_{b}\right)
$$




$$
\bar{\Lambda}_{2, P R}=N^{2} \bar{g} e\left(d_{c} K_{S}+K_{b}\right) .
$$

Furthermore, the variances of $\Lambda_{1, P R}$ and $\Lambda_{2, P R}$ are $N^{2} \sigma_{+, i j}^{2}$ and $N^{2} \sigma_{-, i j}^{2}$, respectively. Assuming that the decision variables obey Gaussian statistics, for a BPPM modulation scheme, the probability of error for the proposed receiver is given by ${ }^{12}$

$$
P_{e}=Q\left(\sqrt{\frac{\left(\bar{\Lambda}_{1, P R}-\bar{\Lambda}_{2, P R}\right)^{2}}{N^{2} \sigma_{+, i j}^{2}+N^{2} \sigma_{-, i j}^{2}}}\right)
$$

where with $Q(x)=\frac{1}{2 \pi} \int_{x}^{\infty} e^{-\frac{y^{2}}{2}} d y$ and it is assumed that we are summing $N^{2}$ decision variables containing the signal to render a decision (the proposed receiver).

Using (10)-(14), we have

$$
P_{e}^{P R}=Q\left(\sqrt{\frac{(\bar{g} e)^{2} N^{4} K_{s}^{2}}{(\bar{g} e)^{2} F\left(N^{2} K_{s}+2 N^{2} K_{b}\right)+2 N^{2} \sigma_{n}^{2}}}\right) .
$$

For a standard receiver, since the location of the beam within the focal plane has not been identified,

$$
P_{e}^{S R}=Q\left(\sqrt{\frac{(\bar{g} e)^{2} N^{4} K_{s}^{2}}{(\bar{g} e)^{2} F\left(N^{2} K_{s}+2 M^{2} K_{b}\right)+2 M^{2} \sigma_{n}^{2}}}\right) .
$$

Note that, for the standard receiver, we consider all $M^{2}$ photodetectors, and hence, the background noise as well as thermal noise contributions are scaled by $M^{2}$.

\subsection{PR Receiver with False Detection}

As noted in the previous work ${ }^{10}$, it is possible that the algorithm used to identify the location of the beam in the focal plane can false lock and identify incorrect set of photodetectors as the candidate detectors in receipt of signal. In that event, the decision variables are merely noise corrupted variables, which in turn will result in an error rate of 0.5 . The noise corrupted photodetectors are receiving background radiation and thermal noise. This further implies that $m_{i j}^{+}=m_{i j}^{-}=\bar{g} e K_{b}$, which results in $50 \%$ error rate. Assuming that the probability of false lock is $P_{F L}$, then the overall bit error rate for the proposed receiver is now

$$
P_{e}^{P R}=\left(1-P_{F L}\right) Q\left(\sqrt{\frac{(\bar{g} e)^{2} N^{4} K_{S}^{2}}{(\bar{g} e)^{2} F\left(N^{2} K_{S}+2 N^{2} K_{b}\right)+2 N^{2} \sigma_{n}^{2}}}\right)+0.5 P_{F L}
$$

Here, we assume that the false lock is an artifact of the search algorithm, which is independent of the signal level. This constitutes the worst-case scenario as the significance of the false lock (shown in the Numerical Results Section) becomes more evident at high signal levels, for which one may expect less frequent false locks. 


\section{NUMERICAL RESULTS}

In this study, we consider $T_{0}=300 \mathrm{~K}$ (room temperature), $R_{L}=50 \Omega, F=1$, and $R_{b}=1$ Gbps. In Figs. $1 \& 2$, we are depicting the bit error rates of the standard and proposed receivers for two levels of background noise. We also assume a gain of $\bar{g}=200$. Furthermore, it is assumed that $P_{F L}=0$.

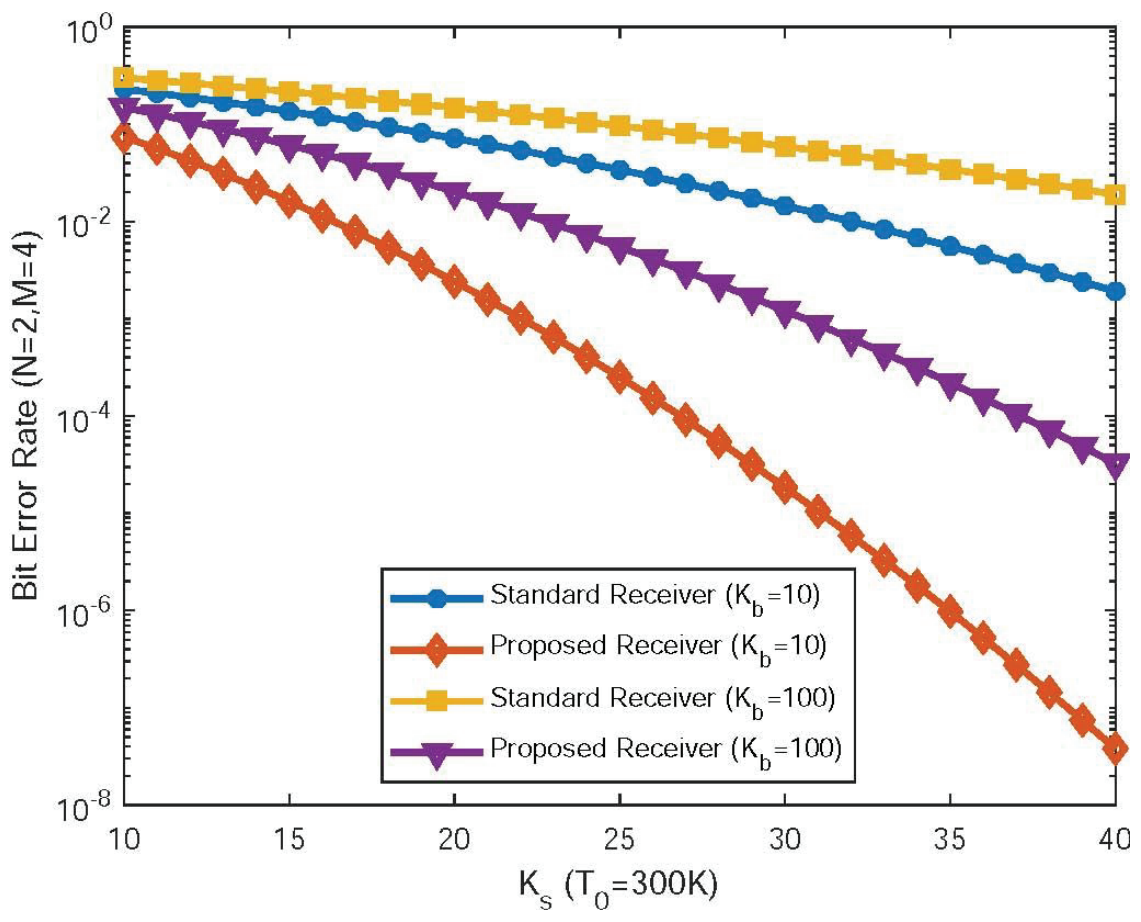

Fig. 1. Bit error rate of the PR and SR for various background noise levels when $\bar{g}=200$ and $P_{F L}=0$.

In Fig. 1 , it is assumed that $N=2$ and $M=4(4 \times 4=16$ detectors), while in Fig. 2 , we have increased $M$ to 10 $(10 \times 10=100$ detectors). It becomes immediately obvious that the proposed receiver yields a performance that is far superior to that of the standard receiver for the parameters considered. As $M$ is increased, due to an increase in the collected background radiation, the standard receiver begins to perform quite poorly. Provided that the bit error rate of the proposed receiver is independent of $M$, we observe no change in the performance of the proposed receiver as $M$ is increased. To gain an insight on the performance of the systems considered here, in Figs. $3 \& 4$, we consider the same scenarios as in Figs. $1 \& 2$, respectively, but when $P_{F L}=10^{-6}$. One obvious implication of this assumption is that the performance of the proposed receiver is lower bounded by a floor at $0.5 \times 10^{-6}$. This is an expected result according to (20). Nonetheless, the proposed receiver outperforms the standard receiver over a wide range of the signal and background noise intensity levels. 


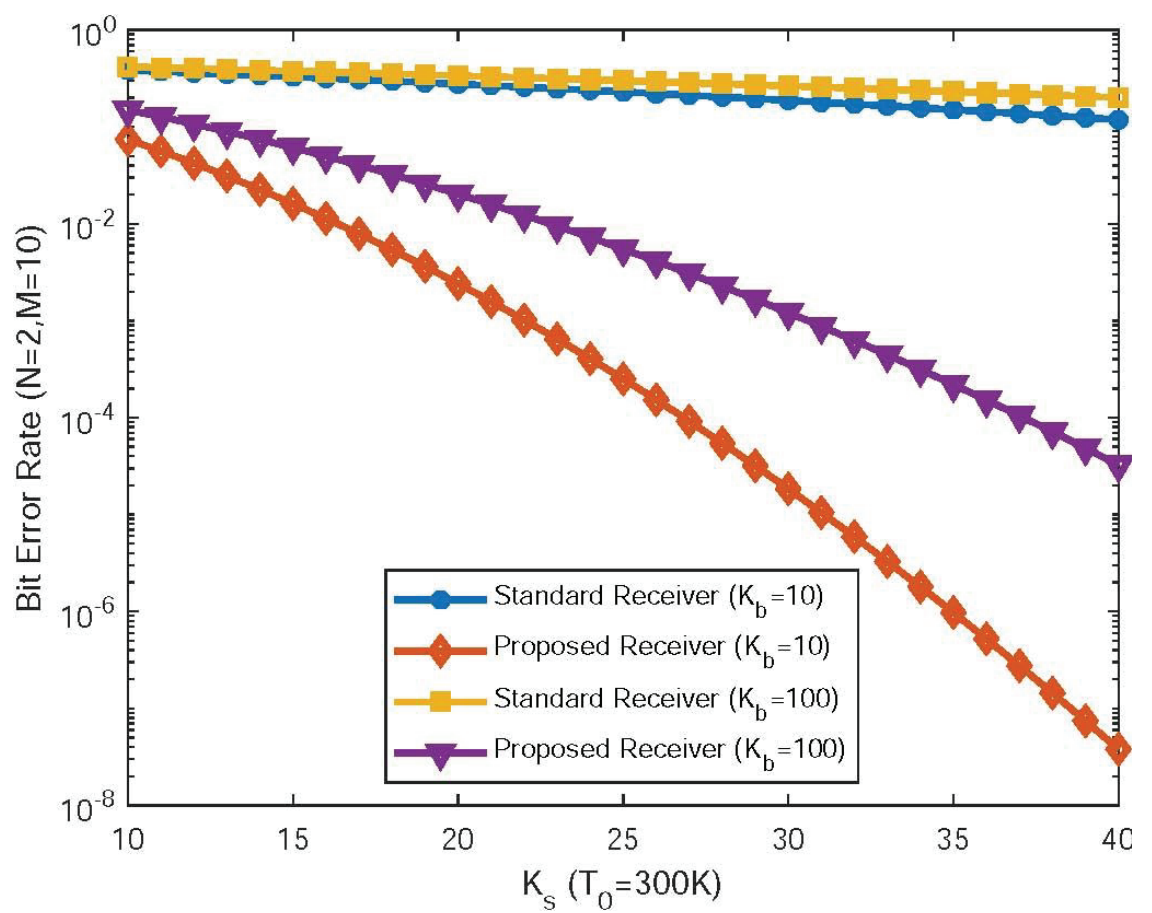

Fig. 2. Bit error rate of the PR and SR for various background noise levels when $\bar{g}=200$ and $P_{F L}=0$.

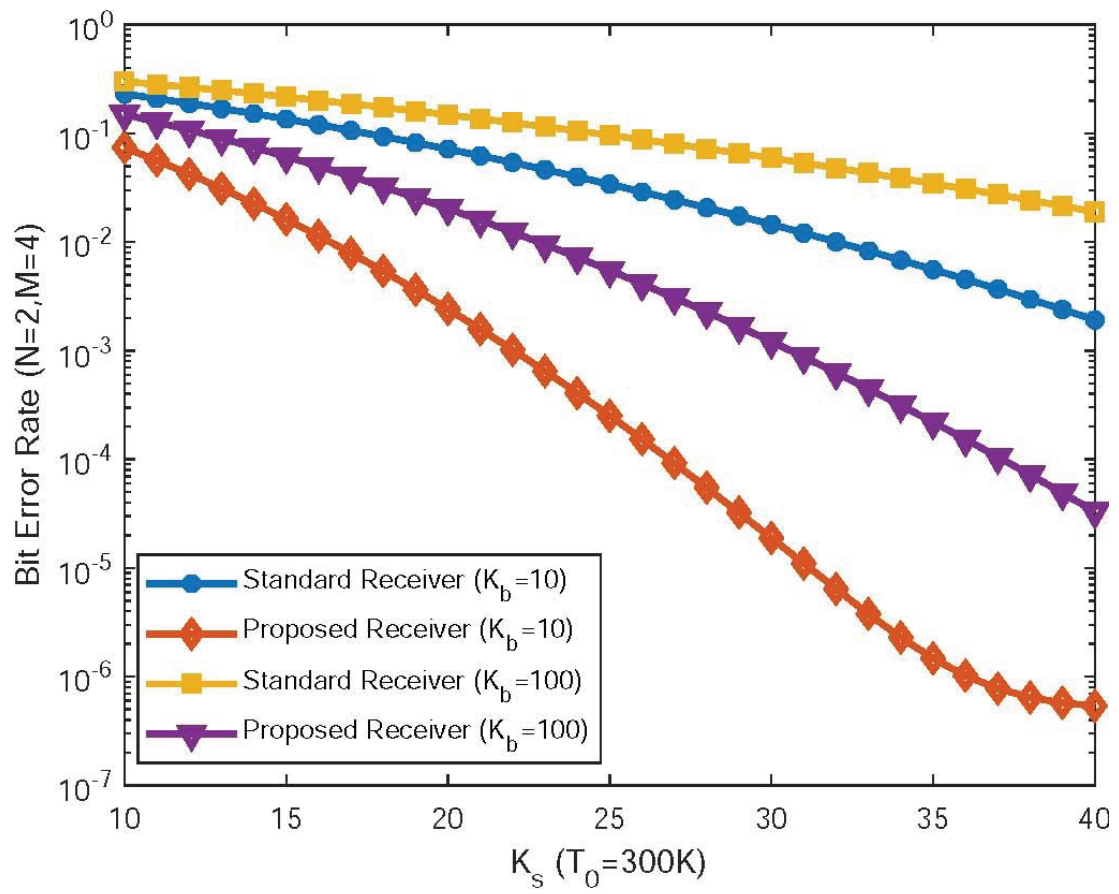

Fig. 3. Bit error rate of the PR and SR for various background noise levels when $\bar{g}=200$ and $P_{F L}=10^{-6}$. 


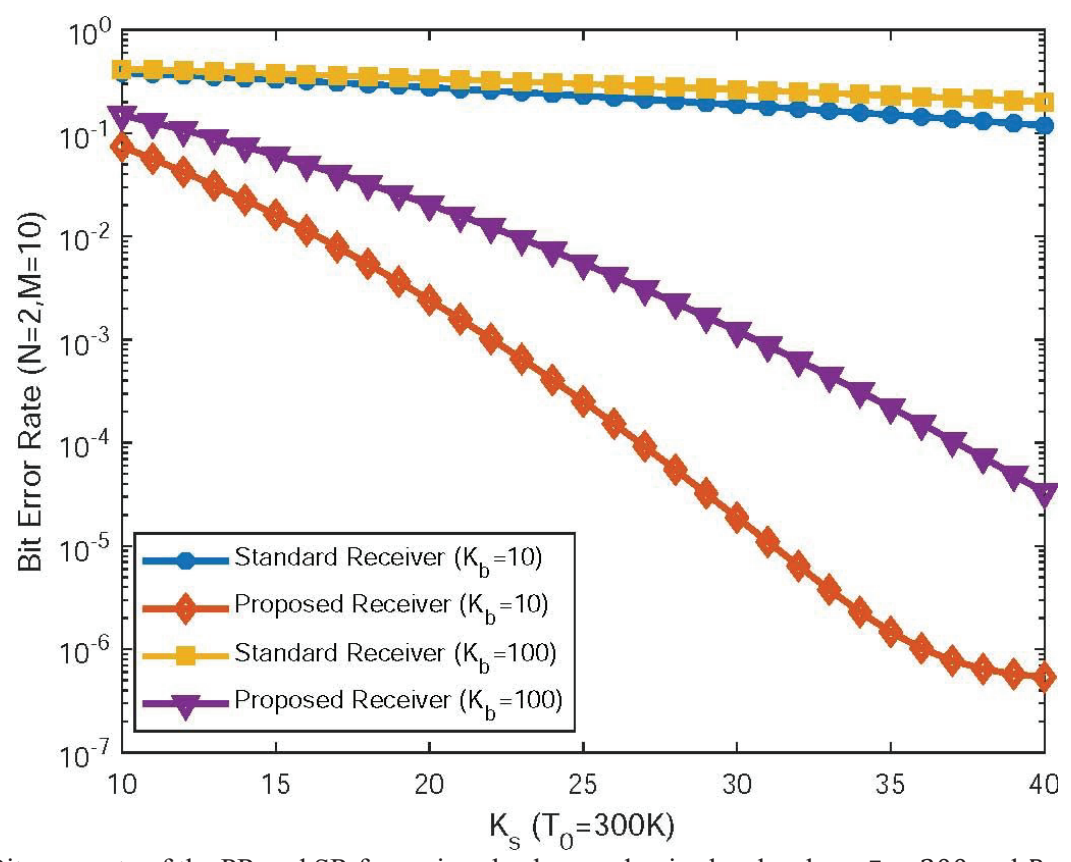

Fig. 4. Bit error rate of the PR and SR for various background noise levels when $\bar{g}=200$ and $P_{F L}=10^{-6}$.

In Figs. $5 \&$, we consider the impact of the average gain of the detector on the performance of the two receivers by reducing $\bar{g}$ from 200 to 50 while keeping all other parameters constant. Two main observations can be made here. First, a significant degradation in performance is observed with the proposed receiver outperforming the standard receiver. Second, the impact of background noise becomes less pronounced. This is due to the fact that a high gain detector increases the impact of background noise while it undermines the impact of thermal noise. For the problem at hand, background noise is a greater impairment than its thermal noise counterpart. For this reason, an increase in the gain will result in a greater change in the performance due to an increase in the background radiation (Figs $3 \& 4$ ). Finally, since the false lock probability is assumed to be $10^{-6}$, its impact is not seen in Figs. $5 \& 6$ as the performance for the signal levels considered here are above this bit error rate floor. In general, for all cases considered here, the performance of the proposed receiver remains superior by several orders of magnitude to that of the standard receiver. Hence, the proposed technique offers a superior alterative to the standard array detection strategy without resorting to the cost- and complexity-prohibitive MRC detection. 


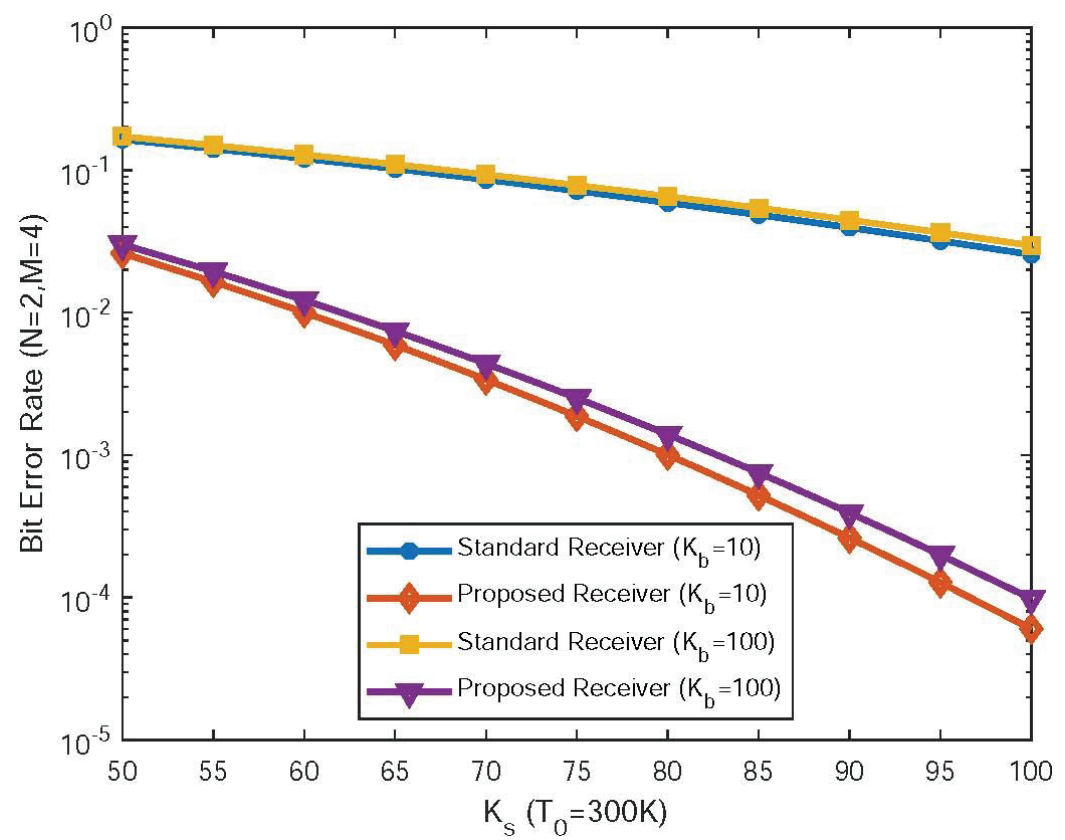

Fig. 5. Bit error rate of the PR and SR for various background noise levels when $\bar{g}=50$ and $P_{F L}=10^{-6}$.

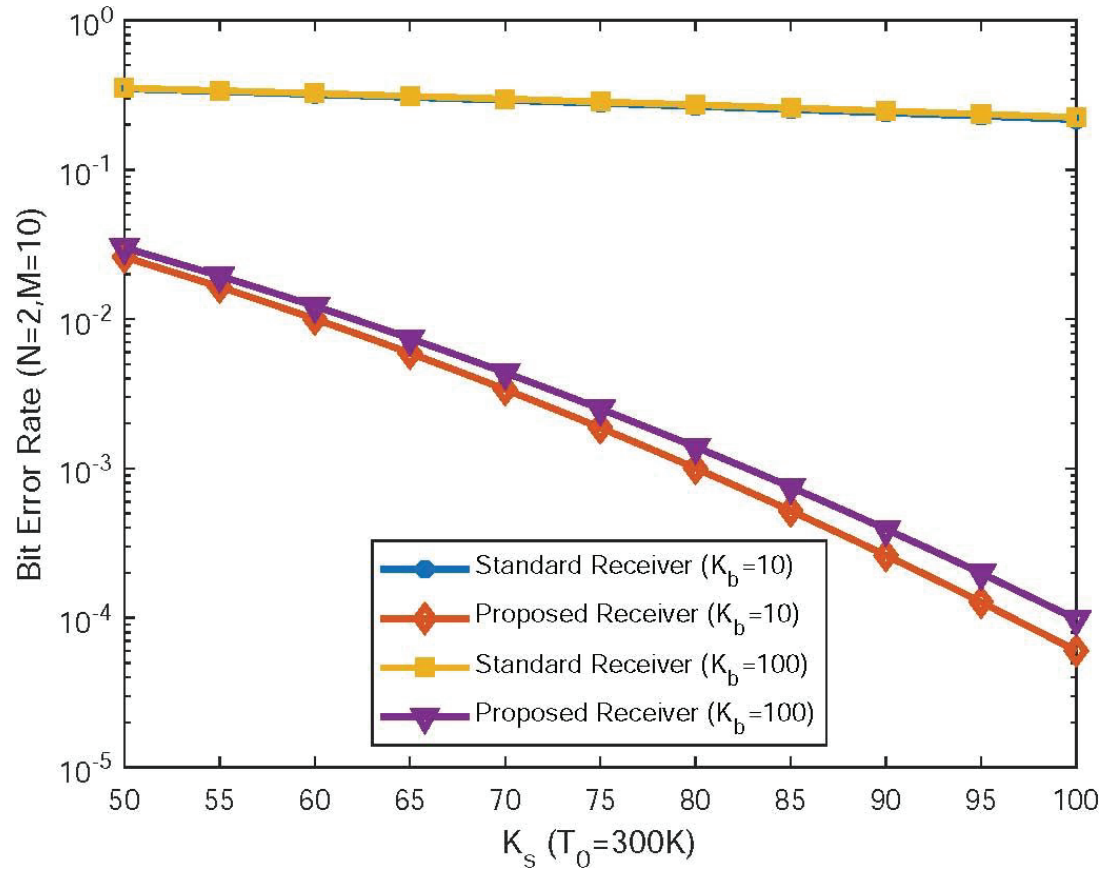

Fig. 6. Bit error rate of the PR and SR for various background noise levels when $\bar{g}=50$ and $P_{F L}=10^{-6}$. 


\section{CONCLUDING REMARKS}

In this paper, an array detection strategy was proposed. The proposed detector took advantage of a recently introduced algorithm for identifying the location of the signal-bearing detectors in a focal plane array receiver. It was shown here that the proposed receiver outperforms the standard array detector by a significant margin over a wide range of background noise levels. Furthermore, it was shown that the proposed receiver performance is independent of the size of the detector array while a standard receiver performance is impacted adversely by an increase in the size of the detector array due to background noise. Therefore, the proposed receiver proved to be a viable detection strategy in background noise limited free space optics communication channels.

\section{REFERENCES}

[1] Mukai, R., Arabshai, P., and Vilnrotter, V. A., “An array feed radial basis function tracking system for NASA's deep space network antennas,” Proc. IEEE-INNS-ENNS International Joint Conference on Neural Networks. IJCNN 2000. Neural Computing: New Challenges and Perspectives for the New Millennium, 259-262 (2000).

[2] Ye, Z, Satorius, E. H., Vilnrotter, V. A., Pham, T. T., and Fort, D. N.," Large antenna array techniques for very low SNR channels," Proc. 2001 MILCOM Proceedings Communications for Network-Centric Operations: Creating the Information Force (Cat. No.01CH37277), 1283-1290 (2001).

[3] Vilnrotter, V. A. and Srinivasan, M., "Adaptive detector arrays for optical communications receivers," IEEE Transactions on Communications, 50(7), 1091-1097 (2002).

[4] Cole, M. and Kiasaleh, K., "Signal estimators for p-i-n and APD-based free-space optical communication systems", Proc. IEEE Global Telecommunications Conference, 2004 (2), 1221-1224 (2004).

[5] Cole, M. and Kiasaleh, K., "Signal intensity estimators for free-space optical communications through turbulent atmosphere", IEEE Photonics Technology Letters, 16(10), 2395-2397 (2004).

[6] Vilnrotter, V. A., Lau, C., Srinivasan, M., Andrews, K., and Mukai, R., "Optical array receiver for communication through atmospheric turbulence," Journal of Lightwave Technology, 23(4), 1664-1675 (2005).

[7] Cole, M. and Kiasaleh, K., "Signal intensity estimators for free-space optical communication with array detectors," IEEE Transactions on Communications, 55(12), 2341-2350 (2007).

[8] Vilnrotter, V. A., Britcliffe, M., and Hoppe, D, "Focal Plane Array Receiver for Deep-Space Communication," Proc. 2008 IEEE Aerospace Conference, 1095-323X (2008).

[9] Kiasaleh, K., "Nonlinear array detection strategies for optical beams in long range free-space optics channels," International Conference on Space Optics - ICSO 2018, edited by Zoran Sodnik, Nikos Karafolas, Bruno Cugny, Proc. of SPIE Vol. 11180, 111805P - (C) 2018 ESA and CNES · CCC code: 0277-786X/18/\$18 · doi: $10.1117 / 12.2536124$

[10] Kiasaleh K., "Agile Optical Receiver with Detector Arrays Optimized using Particle Swarm Optimization” IEEE International Conference on Space Optical Systems and Applications (ICSOS 2019), (Portland, OR), Oct. 14-16 (2019). https://icsos2019.ieee-icsos.org/files/2019/12/ICSOS19 proceedings.zip

[11] Kaur, P., Jain, V. K., Kar, S., "Performance analysis of FSO array receivers in presence of atmospheric turbulence," IEEE Photonics Technology Letters, 26(12), 1165-1168 (2014).

[12] Gagliardi, R. M. and Karp, S., [Optical Communications], Wiley Series in Telecommunications and Signal Processing, New York, 119-146 (1995). 